\title{
The development of a conditioned positive reinforcer based upon the termination of shock'
}

\author{
William Wagman and Joseph D. Allen \\ SOUTHERN ILLINOIS UNIVERSITY
}

\begin{abstract}
The present study was an attempt to clarify the data concerned with the adient properties of stimuli which have been associated with the termination of shock. Six different groups of rats were subjected to varying numbers of shock-tone pairings. The positively reinforcing effects of the tone were tested in an operant conditioning situation. The data showed that these properties are demonstrated within a narrow range of shock-tone pairings.
\end{abstract}

\section{Introduetion}

Current reinforcement theories (Keller \& Schoenfeld, 1950; Mowrer, 1960) hypothesize that the termination of shock is a positive reinforcer. It should than follow that stimuli associated with this event develop positively reinforcing properties. The research on this latter point, however, has produced contradictory results. Barlow (1952) and Evans (1962), for example, have reported the successful development of a positive secondary reinforcer while several studies have failed to demonstrate this phenomenon (Beck, 1961). The reason for these contradictory results appears to be the number of shock-tone pairings that have been used. The unsuccessful studies have employed 25 to 100 pairings; the successful, one to eight. The present study was designed to investigate this parameter. Method

Forty-five male rats of albino, hooded and Harvard Brown strains were divided into nine groups of five Ss each. The groups contained the same proportion of animals in each strain. The procedure involved three stages. Initially, all subjects were trained to press a lever on a CRF schedule in an operant conditioning test chamber for $45 \mathrm{mg}$ food pellets and remained in the chamber for $1 \mathrm{hr}$. or until 100 reinforcements were received. Most animals met this requirement. On the third day all animals were again placed in the operant box for a 30-min. period of free responding on $\mathrm{CRF}$. The number of responses made during this period was used as a measure of the subject's pre-test response strength.

Stage II was initiated on the fourth day. The subjects were placed in a shock chamber where they were exposed to shock-tone pairings. At this point they were split into six experimental and three control groups. The experimental groups all received 1 ma shocks (3-sec. duration) followed by a $.5 \mathrm{sec} .6000$ cycle tone. Each of the experimental groups received a different number of shock-tone pairings $(2,4,8,12,16$, and 20$)$. These stimuli were presented on a VI schedule for a

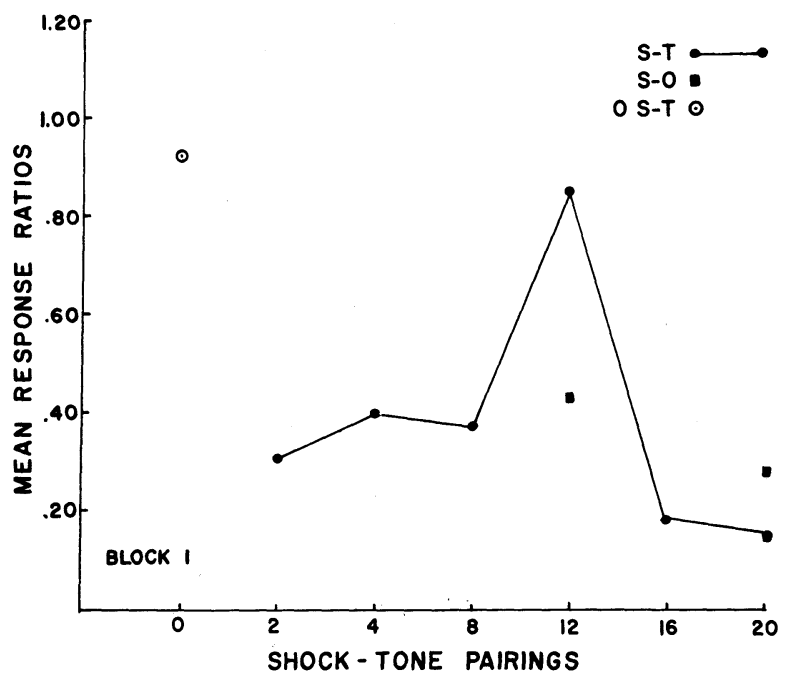

Fig. 1. Mean response ratios for all subjects during test phase. Each point represents the mean of five subjects. The $\mathrm{S}-\mathrm{T}$ groups are the experimental subjects. The S - O, the two no-tone controls and the OS $\mathrm{T}$, the group which received neither shock nor tone.

1-hr. period. The schedule was designed so that the minimum time between stimuli was $2 \mathrm{~min}$. Two of the control groups received 12 and 20 presentations of shock with no tone. The third group was put into the shock chamber but no shocks or tones were delivered.

The test phase of the experiment began $23 \mathrm{hr}$. after the shock exposures. All subjects were put into the operant conditioning chamber for $1 \mathrm{hr}$. During this period each bar-press response produced the tone. The number of responses for successive 10-min. periods was recorded.

\section{Results and Discussion}

Response measures for each animal were converted into ratio scores. These scores represented the number of responses made during the first $30-\mathrm{min}$. period of the 1-hr. post-test session divided by the number of responses made during the pre-test session. Figure 1 illustrates these ratio scores as a function of the number of shock-tone pairings during the first $30 \mathrm{~min}$. of the test session. The response ratios rose to a maximum value at 12 pairings and declined rapidly at the higher numbers of shock-tone pairings. This showed that the responses in the test situation were differentially reinforced over the range of shock-tone pairings. The difference between the 12-pairing group and its control was statistically significant $(p<.01)$. The 20 -pairing 
group was not significantly different from its control but was significantly different from the 12-pairing group $(\mathrm{p}<.01)$. The 12-pairing group, however, did not differ from the zero-shock group, but did differ from the 16-pairing group $(p<.01)$ and from the twopairing group $(p<.05)$. Student's $t$ was used in the initial test; Tuckey tests (Winer, 1961) were used in all other tests.

The data indicate that stimuli which are associated with the termination of shock develop conditioned reinforcing properties. The degree of reinforcing influence appears to be limited by the suppressive nature of other stimuli which are associated with the shock. This suppression is illustrated by the three control groups. The exposure to the shock significantly lowered the number of responses to extinction in situations in which the tone had never been associated with the shock.

The present data can be explained as a balance between the reinforcing and suppressive properties of the stimuli which have been associated with the shock. There is a critical number of pairings which will permit maximal expression of the positively reinforcing effects of the tone. Below this level the suppressive effects of shock are strong and the positively reinforcing effects of shock termination are not well-established. Above this level the noxious effects of the shock experience accumulate faster than the adient effects of its termination. This may explain the apparently contradictory results of the previous studies in thia area.

\section{References}

BARLOW, J. A. Secondary motivation through classical conditioning: a one-trial non-motor learning in the white rat. Amer. Psychol., 1952, 7, 273.

BECK, R. C. On secondary reinforcement and shock termination. Psychol. Bull., 1961, 58, 28-45.

EVANS, W. O. Producing either positive or negative tendencies to a stimulus associated with shock. J. exp. Anal. Beh., 1962, 5, 335-337.

KELLER, F. S., \& SCHOENFELD, W. N. Principles of Psychology. New York: Appleton-CenturyCrofts, 1950.

MOWRER, O. H. L e a rning Theory and B e havior. New York: Wiley, 1960.

WINER, B. O. Statistical Principles in Experimental Design. New York: McGraw-Hill, 1962.

\section{Note}

1. This study was partially supported by grant no. 17155 from the Illinois Psychiatric Training and Research Authority and NIMH grant no. MH10463-01. 